\title{
Functional analysis and applications
}

by Grégoire Allaire

Basic course of the Master Program "Mathematical Modelling" Ecole Polytechnique and Pierre et Marie Curie University Academic year 2015-2016

\section{Introduction}

\subsection{Motivation: pde's and optimization}

\subsection{Banach spaces}

English: section 3.1 in [3].

\subsection{Hilbert spaces}

French: sections V.1 of [1]. English: sections 5.1 of [2], section 4.1 in [3].

\subsection{Examples: Lebesgue $L^{p}$ spaces}

French: sections IV.1 and IV.2 of [1]. English: sections 4.1 and 4.2 of [2], section 3.4 in [3].

Measurable functions, simple functions, Young's inequality, Hölder inequality, completeness of $L^{p}$, norm convergence versus almost everywhere convergence.

\section{Linear operators on Banach spaces}

\subsection{Definitions}

Linear and continuous applications, duality, bidual. Dual space of $L^{p}$, HahnBanach theorem.

English: section 3.5 in [3].

\subsection{The "great" theorems of Banach}

Banach-Steinhaus theorem, open mapping theorem, closed graph theorem.

French: sections II.1, II.2 and II.3 of [1]. English: sections 2.1, 2.2 and 2.3 of [2], sections 5.3, 5.6 and 5.7 in [3]. 


\subsection{Introduction to unbounded operators}

Domain, adjoint

French: section II.6 of [1]. English: section 2.6 of [2].

\section{Weak convergence}

\subsection{Definitions}

Weak and weak-* sequential convergence.

French: section III.2 of [1]. English: section 3.2 of [2], section 5.12 in [3].

\subsection{Sequential compactness}

Proof of sequential weak-* compactness (by a diagonal sequence extraction). Statement of sequential weak compactness. Weak lower semi-continuity of convex functionals.

French: sections III.4, III.5 and III.6 of [1]. English: sections 3.4, 3.5 and 3.6 of [2].

\subsection{A few words about topology}

Link with the notion of topological space and definitions of weak topologies.

\subsection{Application to Lebesgue $L^{p}$ spaces}

Examples (oscillation, concentration, escape at infinity, evanescence).

French: section IV.3 of [1]. English: section 4.3 of [2].

\section{Compact operators and spectral analysis}

\subsection{Compact operators and Fredholm alternative}

Definition of compact operators in a Hilbert space. Riesz lemma. Fredholm alternative in a Hilbert space. Application to integral equations.

French: sections V1.1 and VI.2 of [1]. English: sections 6.1 and 6.2 of [2]. 


\subsection{Self-adjoint operators}

Definition of self-adjoint operators in a Hilbert space. Eigenvalues and eigenfunctions. Spectral decomposition of self-adjoint compact operators.

French: section VI.4 of [1]. English: section 6.4 of [2].

\section{Distributions, Fourier analysis and Sobolev spaces}

\subsection{Distributions}

Definition, convergence of test functions in $C_{c}^{\infty}(\Omega)$, convergence of distributions

French: chapter 3 in [4]. English: section 6.3 in [3].

\subsection{Fourier analysis}

Definition in the Schwartz class and in $L^{2}\left(\mathbb{R}^{N}\right)$

French: chapter 9 of [5]. English: chapter 9 in [9].

\subsection{Sobolev spaces}

Definition of $W^{m, p}(\Omega)$ for an integer $m \in \mathbb{N}$. Definition of $H^{s}\left(\mathbb{R}^{N}\right)$ for a real $s \geq 0$. Definition of a $C^{1}$ smooth open set. Extension operator, partition of unity and the local charts argument. Non-local norm on $W^{s, p}(\Omega)$ for $0<s<1$ and its equivalence with the Fourier definition for $p=2$. Negative Sobolev spaces as duals of $W_{0}^{m, p}(\Omega)$.

French: section IX.1 of [1]. English: section 9.1 of [2].

\subsection{Embeddings and traces}

Trace theorem from $H^{1}(\Omega)$ into $H^{1 / 2}(\partial \Omega)$ (with proof by partition of unity and Fourier analysis). Sobolev embedding theorems (with proof in $\mathbb{R}^{N}$ ).

French: section IX.3 of [1]. English: section 9.3 of [2].

\subsection{Compactness}

Rellich and Rellich-Kondrachov theorems (with a proof by Fourier series in the torus).

French: section IX.3 of [1]. English: section 9.3 of [2]. 


\subsection{Time dependent Sobolev spaces}

Definition of $L^{p}\left((0, T) ; W^{m, q}(\Omega)\right)$ and $C\left((0, T) ; W^{m, q}(\Omega)\right)$. Aubin-Lions compactness lemma.

French: volume 1, chapter 3 of [6]. English: volume 1, chapter 3 of [7].

\section{References}

[1] H. Brézis Analyse fonctionnelle, Masson, Paris (1983).

[2] H. Brézis Functional analysis, Sobolev spaces and partial differential equations, Universitext. Springer, New York (2011).

[3] Ph. Ciarlet, Linear and Nonlinear Functional Analysis with Applications, SIAM, Philadelphia (2013).

[4] F. Golse, Distributions, analyse de Fourier, équations aux dérivées partielles, Ecole Polytechnique (2011).

[5] F. Golse, Y. Laszlo, F. Pacard, C. Viterbo, Analyse réelle et complexe, Ecole Polytechnique (2013).

[6] J.-L. Lions, E. Magenes, Problèmes aux limites non homogènes et applications, Travaux et Recherches Mathmatiques, No. 17 Dunod, Paris 1968.

[7] J.-L. Lions, E. Magenes, Non-homogeneous boundary value problems and applications, Die Grundlehren der mathematischen Wissenschaften, Band 181. Springer-Verlag, New York-Heidelberg, 1972.

[8] W. McLean, Strongly elliptic systems and boundary integral equations, Cambridge University Press, Cambridge, 2000.

[9] W. Rudin, Real and complex analysis, Third edition. McGraw-Hill Book Co., New York (1987).

[10] L. Tartar, An introduction to Sobolev spaces and interpolation spaces, Lecture Notes of the Unione Matematica Italiana, 3. Springer, Berlin; UMI, Bologna, 2007. 